\title{
The Approximate Analytical Solution of Non-Linear Equation for Simultaneous Internal Mass and Heat Diffusion Effects
}

\author{
Mayathevar Renugadevi', Saminathan Sevukaperumal ${ }^{2}$, Lakshmanan Rajendran ${ }^{3 *}$ \\ ${ }^{1}$ Department of Mathematics, P. M. T. College, Usilampatti, India \\ ${ }^{2}$ Department of Mathematics, J. J. College of Arts and Science, Pudukkottai, India \\ ${ }^{3}$ Department of Mathematics, Sethu Institute of Technology, Kariapatti, India

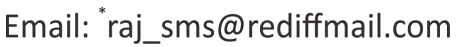

Received 17 May 2016; accepted 26 June 2016; published 29 June 2016

Copyright (C) 2016 by authors and Scientific Research Publishing Inc.

This work is licensed under the Creative Commons Attribution International License (CC BY). http://creativecommons.org/licenses/by/4.0/

CC (i) Open Access

\begin{abstract}
For the first time a mathematical modelling of porous catalyst particles subject to both internal mass concentration gradients as well as temperature gradients, in endothermic or exothermic reactions has been reported. This model contains a non-linear mass balance equation which is related to rate expression. This paper presents an approximate analytical method (Modified Adomian decomposition method) to solve the non-linear differential equations for chemical kinetics with diffusion effects. A simple and closed form of expressions pertaining to substrate concentration and utilization factor is presented for all value of diffusion parameters. These analytical results are compared with numerical results and found to be in good agreement.
\end{abstract}

\section{Keywords}

Chemical and Biological Systems, Modified Adomian Decomposition Method, Nonlinear Reaction Diffusion, Porous Catalyst Particles, Mass and Diffusion Effect

\section{Introduction}

In many engineering and industrial applications, catalytic processes in chemical reactors are often considered to be very useful. This induces particular attention to the study of catalytic reactions at the single-particle level [1]. Moreover, the reaction behavior of porous catalyst particles had been studied over nearly a quarter of a century [2]-[4]. Majority of chemical reactions are accompanied by heat transfer effects; they either release or absorb

\footnotetext{
${ }^{*}$ Corresponding author.
}

How to cite this paper: Renugadevi, M., Sevukaperumal, S. and Rajendran, L. (2016) The Approximate Analytical Solution of Non-Linear Equation for Simultaneous Internal Mass and Heat Diffusion Effects. Natural Science, 8, 284-294. 
heat. This can lead to appreciable increase (or decrease) of temperature toward the particle centre [5]-[7]. Since chemical reaction rates vary rapidly increase with temperature, this effect could radically change the behavior of the catalyst particles. Analysis of chemical kinetics with diffusion effects usually leads to solving strongly nonlinear differential equations. Detailed reviews of mathematical models describing reactions in porous catalyst particle can be found in [8]. Assuming a flat geometry for the particle and that conductive heat transfer is negligible compared to convective heat transfer. The approximate behavior of the functional forms is sufficiently similar for various geometric forms [9] [10] so that the spherical particle is a approximation [11] [12] for most cases encountered, such as cylindrical pellets, or irregular granules. When the chemical reaction is accompanied by a heat effect, not only a mass concentration gradient, but also appreciable temperature gradients can exist within the particle. Weisz and Hicks [13] solved the non-linear mass balance equation using numerical method.

However, to the best of our knowledge, there was no rigorous analytical solution for the concentration of reactant of catalyst having been reported. The purpose of this communication is to derive simple analytical expression for concentration and utilization factor for all possible values of reaction/diffusion parameters using the modified Adomian decomposition method.

\section{Mathematical Formulation of the Problem}

The dimensionless mass transport equation of porous catalyst particle is [13]

$$
\frac{\mathrm{d}^{2} y}{\mathrm{~d} x^{2}}+\frac{2}{x} \frac{\mathrm{d} y}{\mathrm{~d} x}=\phi_{0}^{2} y \exp \left(\gamma \beta \frac{1-y}{1+\beta(1-y)}\right)
$$

where

$$
\begin{gathered}
y=\frac{c}{c_{0}}, \quad x=\frac{r}{R}, \quad \phi_{0}=R \sqrt{\frac{k_{0}}{D}}, \quad \beta=\frac{c_{0} H D}{K T_{0}}=\left(\frac{\Delta T}{T_{0}}\right)_{\max } \\
\Delta T=T-T_{0}=-\frac{H D}{K}\left(c_{0}-c\right), \quad \gamma=\frac{Q}{R T_{0}}
\end{gathered}
$$

where $y$ is the dimensionless concentration, $x$ is the dimensionless radius of the spherical catalyst pellet, $c$ is the dimensionless concentration of reactant, $K$ is thermal conductivity, $H$ is molar heat of reaction. The parameter $\gamma$ expresses the sensitivity of the reaction rate to temperature; $\beta$ is the maximum temperature variation $(\Delta T)_{\max }$ which could exist within the particle relative to the boundary temperature. The boundary conditions are

$$
y(1)=1,\left(\frac{\mathrm{d} y}{\mathrm{~d} x}\right)_{x=0}=0
$$

The utilization factor $(\eta)$ is given by

$$
\eta=\frac{3}{\phi_{0}^{2}}\left(\frac{\mathrm{d} y}{\mathrm{~d} x}\right)_{x=1}
$$

\section{Analytical Expression of the Concentration Using Modified Adomian Decomposition Method (MADM)}

In the recent years, much attention is devoted to the application of the Adomian decomposition method to the solution of various scientific models [14]. The MADM yields, without linearization, perturbation, transformation or discretisation, an analytical solution in terms of a rapidly convergent infinite power series with easily computable terms. The decomposition method is simple and easy to use and produces reliable results with few iterations. The rate of convergence of modified Adomian decomposition method is higher than standard Adomian decomposition method [15]-[17]. Using this method (see Appendix A), we can obtain the analytical expression of concentration (see Appendix B), of the substrate as follows:

$$
y(x)=1-\frac{\phi_{0}^{2}}{6}+\frac{7}{360} \phi_{0}^{4}(1-\gamma \beta)+\phi_{0}^{2}\left(\frac{1}{6}-\frac{\phi_{0}^{2}(1-\gamma \beta)}{36}\right) x^{2}+\frac{\phi_{0}^{4}(1-\gamma \beta)}{120} x^{4}
$$


Using Equations (5) and (6), we can obtain the effectiveness factor

$$
\eta=1-\frac{\phi_{0}^{2}(1-\gamma \beta)}{15}
$$

The Equation (6) and (7) represent the new and simple analytical expression of concentration of substrate and effectiveness factor provided

$$
\frac{\phi_{0}^{2}}{6}-\frac{7}{360} \phi_{0}^{2}(1-\gamma \beta)<1 \text { and } \frac{\phi_{0}^{2}(1-\gamma \beta)}{15}<1
$$

\section{Numerical Simulation}

The diffusion Equation (1) for the boundary condition (Equation (4)) is also solved numerically. We have used the function pdex1 in MATLAB software to solve numerically the initial-boundary value problem for the nonlinear differential equation. This numerical solution is compared with our analytical results in Figure 1 and Figure 2. Upon comparison, it gives a satisfactory agreement for all values of the dimensionless parameters $\gamma, \beta$ and $\phi$. The Matlab program is also given in Appendix $C$.

\section{Discussion}

The nonlinear system for coupled heat and mass transfer in a spherical non-isothermal catalyst pellet is solved analytically. The concentration of substrate depends on the following three factors $\gamma, \beta$ and $\phi_{0} \cdot \gamma$ is the activation energy parameter and $\beta$ is the heat of reaction parameter which represents the ratio of the characteristic time of the enzymatic reaction to that of substrate diffusion.

Figure 1(a) and Figure 1(b) show the dimensionless concentration of substrate $y$ for various dimensionless pellet raidus $x$. The concentrations were computed for various values of the dimensionless parameter. From Figure 1(a) and Figure 1(b), it is evident that the value of concentration $y \approx 1$ when $x=1$ and $\phi \leq 0.5$ for all values of $\gamma$ and $\beta$. The concentration differs significantly for all values of parameters $\gamma$ and $\beta$. The value of the concentration $y$ decreases when $\phi_{0}$ increases.

The normalized numerical simulation of three dimensional substrate concentration $y$ versus dimensionless pellet radius $x$ is shown in Figures 2(a)-(c). The time independent concentration $y$ is represented in Figures 2(a)-(c). For fixed value of $\beta=0.1$, concentration $y(x)$ is slowly decreasing when $\phi$ is increasing. Then

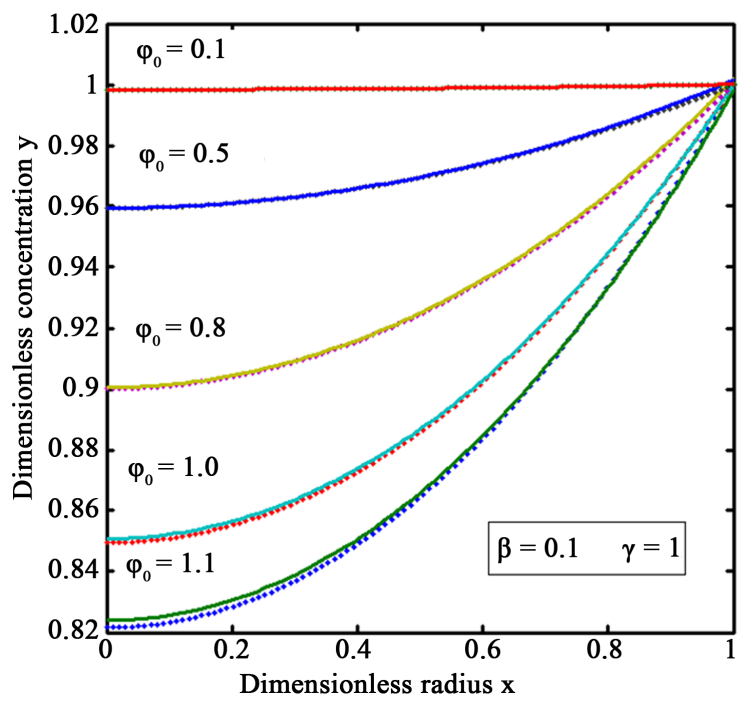

(a)

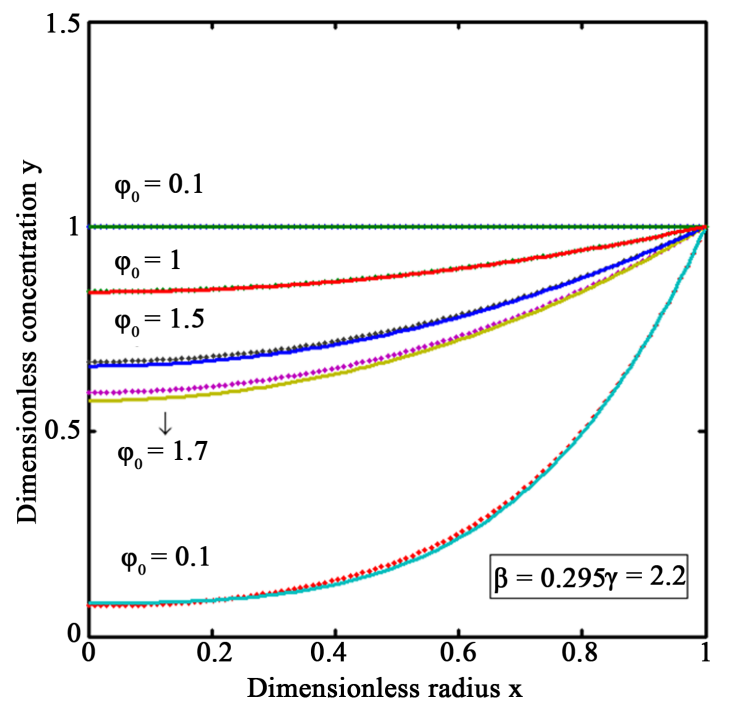

(b)

Figure 1. Plot of dimensionless concentration $y$ versus dimensionless pellet radius $x$. The concentrations were computed for various values of the dimensionless parameter $\phi$ when (a) $\beta=0.1, \gamma=1$ (b) $\beta=0.295, \gamma=2.2$. The curves are plotted using Equation (6). ( - ) denotes the analytical results and ( $\cdots$ ) denotes the numerical simulations. 


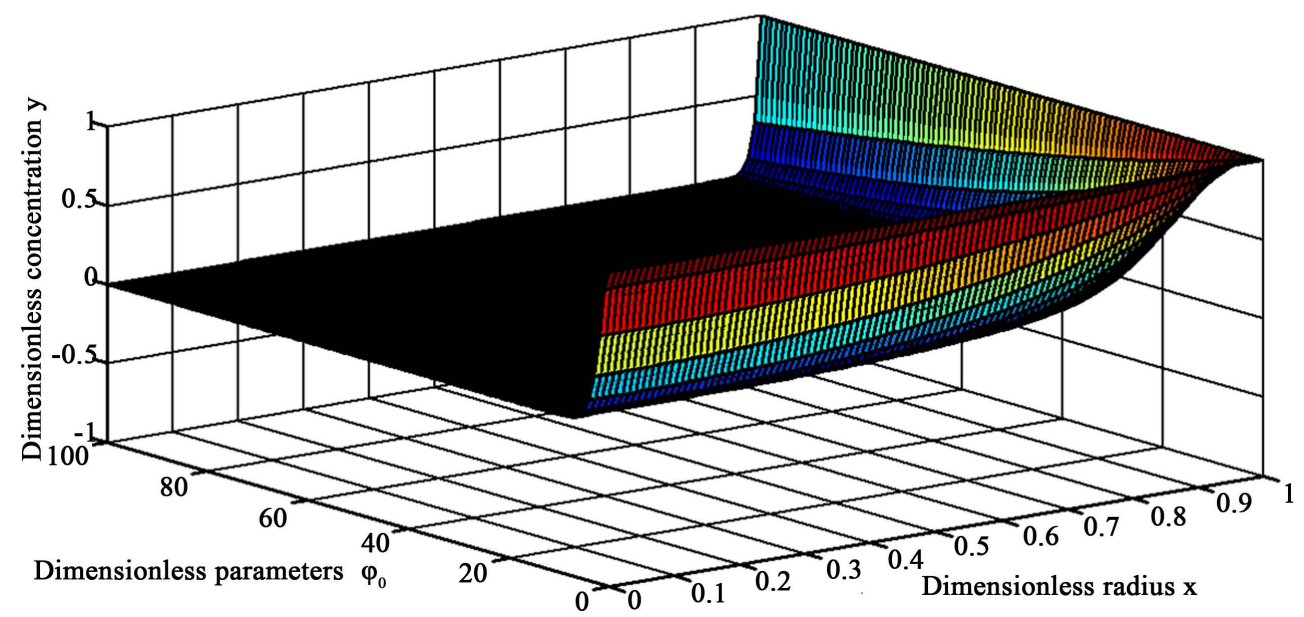

(a)

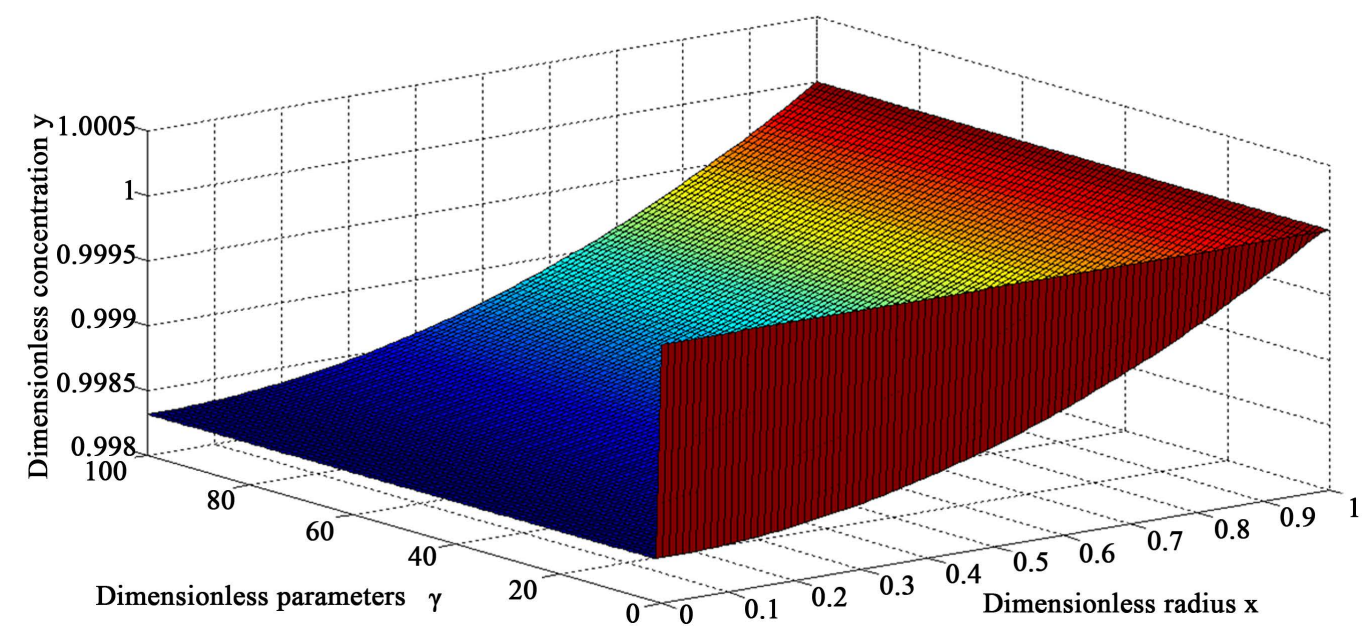

(b)

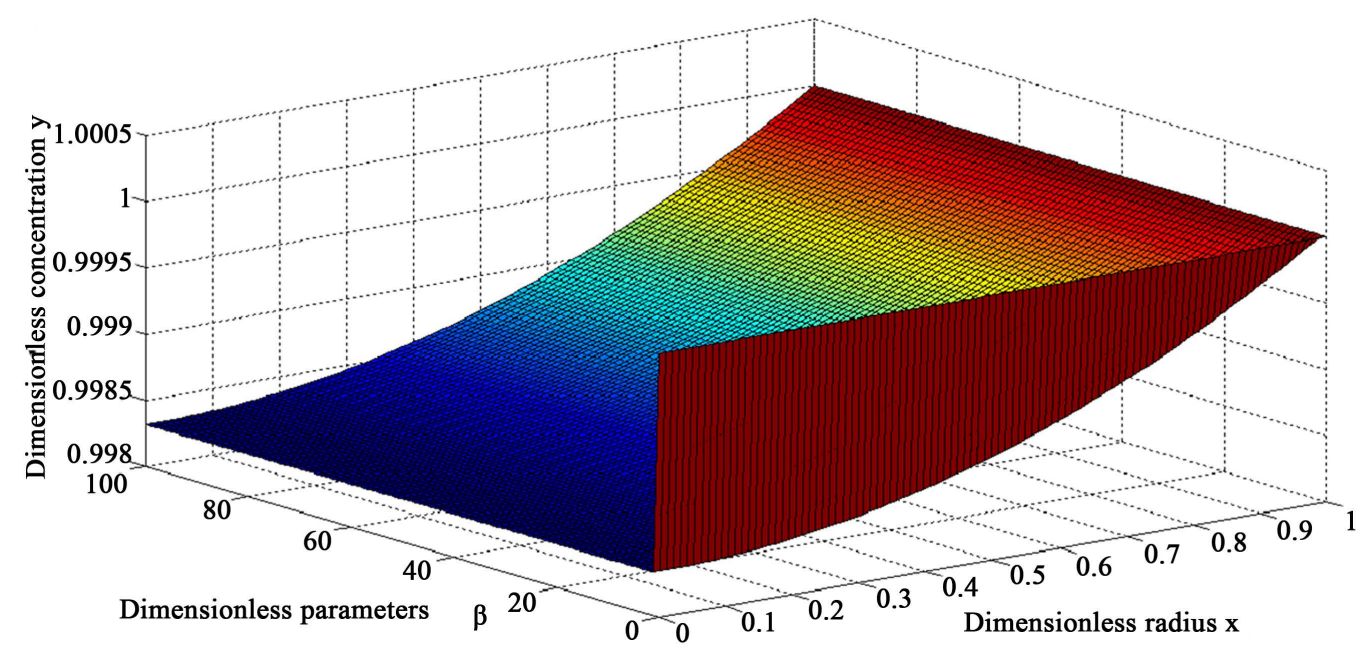

(c)

Figure 2. The normalized dimensionless concentration $y$ versus dimensionless pellet radius $x$ and dimensionless parameters $\phi_{0}, \gamma$ and $\beta$ calculated using Equation (6). The plot was constructed for the values of (a) $\beta=0.1, \gamma=0.1$, (b) $\phi=0.1, \beta=0.1$ and (c) $\phi=0.1, \gamma=0.1$. 
the concentration of $y(x)=1$ when $x=1$ and also for all values of $\phi_{0}, \gamma$ and $\beta$. From these figure, it should be noted that the value of the concentration of substrate decreases for all values of $\gamma$. From this Figures, it is apparent that the value of the concentration of substrate increases when the values of $\beta$ increases.

The variation in effectiveness factor for various values of $\gamma, \beta$ and $\phi_{0}$ using Equation (7) is shown in Figure 3 and Figure 4. From Figure 3, it is evident that the effectiveness factor increases with the increasing value of the dimensionless parameter $\gamma$. From Figure 4, it is also observed that the effectiveness factor increases with the increasing value of the dimensionless parameter $\beta$.

\section{Conclusion}

In this work, we have discussed the mathematical model of catalyst particle in a porous medium through which reactants diffuses. We have obtained the approximate analytical expression for the steady state concentration of substrate for all values of $\gamma$ and $\beta$ in a packed bed reactor using the modified Adomian decomposition method. A satisfactory agreement with the numerical result is noted. Moreover, we have also presented a closed form expression for the utilization factor. The proposed model can be used to solve the nonlinear convective mass and heat diffusion problems.

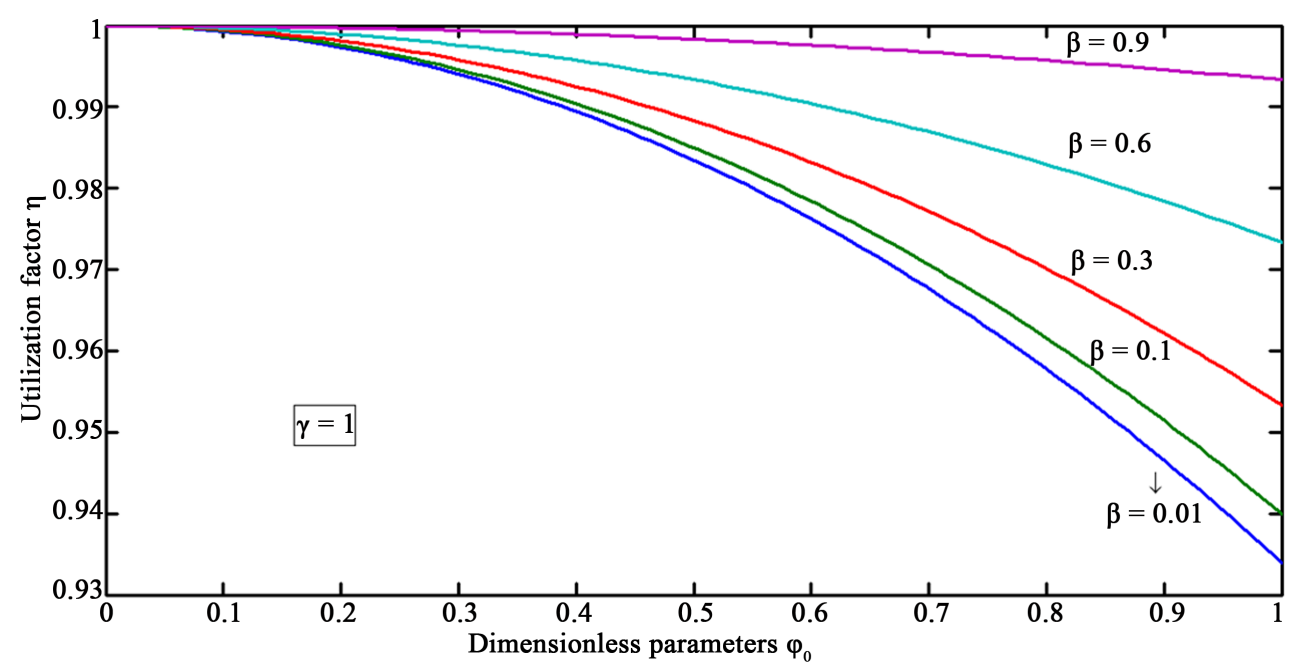

(a)

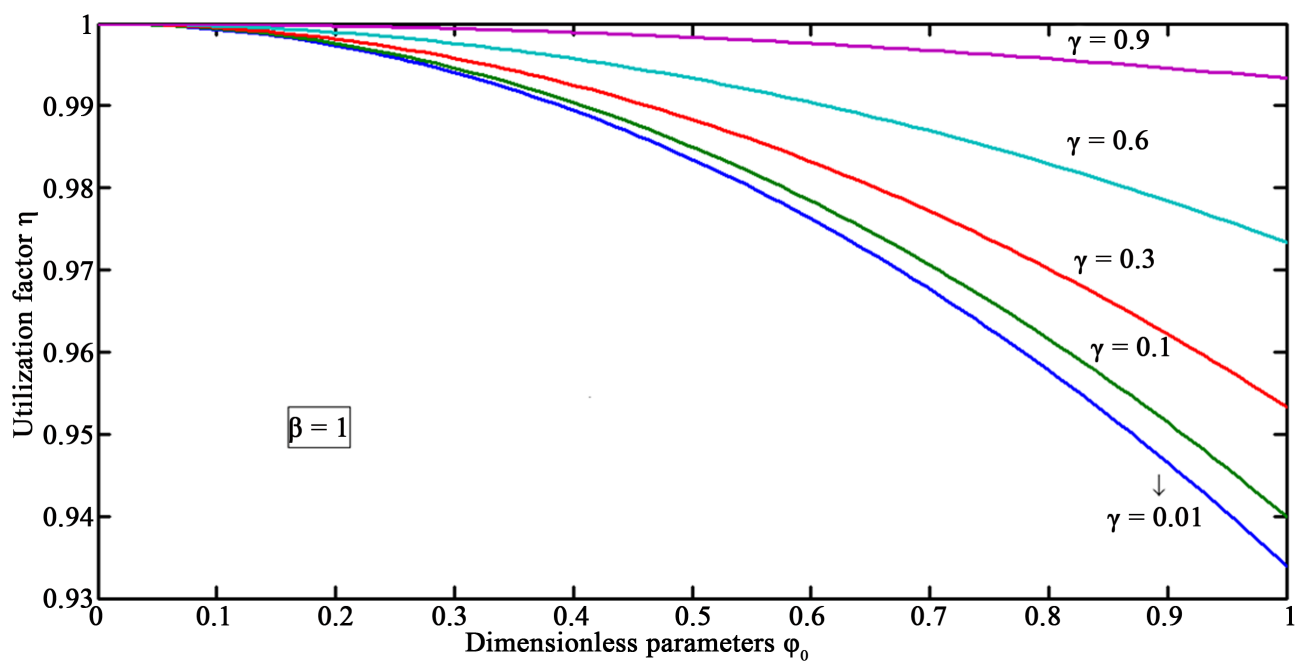

(b)

Figure 3. Plot of the utilization factor $\eta$ versus dimensionless parameter $\phi_{0}$ for various values of (a) $\beta$ and (b) $\gamma$. 


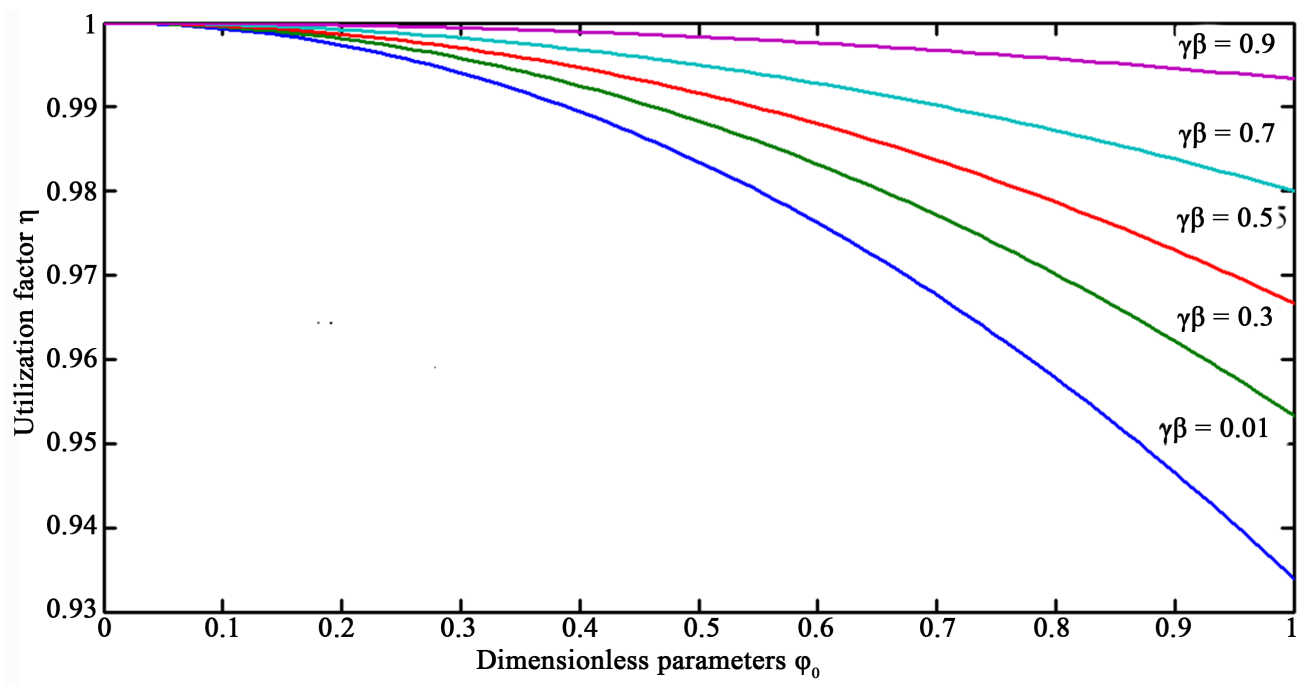

Figure 4. Plot of the utilization factor $\eta$ versus dimensionless parameter $\phi_{0}$ for various values of $\gamma \beta$.

\section{Acknowledgements}

The authors express their gratitude to the reviewers for their valuable comments to improve the quality of the manuscript. This work was supported by the Department of Science and Technology (DST) (No. SB/SI/PC50/2012), New Delhi, India. The authors are thankful to the Head of the Department of Mathematics, Principal and Chairman of Sethu Institute of Technology, Kariapatti for their encouragement.

\section{References}

[1] Kierzenka, J. and Shampine, L.F. (2001) A BVP Solver Based on Residual Control and the MATLAB PSE. ACM Transactions on Mathematical Software, 27, 299-316.

[2] Damkohler, G. (1943) Ubertemperatur in kontaktkornern (Excess Temperature in Catalyst Grains). Zeitschrift f"ur Physikalische Chemie, 193, 16-28.

[3] Kim, D.N. and Kim, Y.G. (1981) An Experimental Study of Multiple Steady States in a Porouscatalyst Due to Phase Transition. Journal of Chemical Engineering of Japan, 14, 311-317. http://dx.doi.org/10.1252/jcej.14.311

[4] Kim, D.H. and Kim, Y.G. (1981) Simulation of Multiple Steady States in a Porous Catalyst Due to Phase Transition. Journal of Chemical Engineering of Japan, 14, 318-322. http://dx.doi.org/10.1252/jcej.14.318

[5] Jaguste, D.N. and Bhatia, S.K. (1991) Partial Internal Wetting of Catalyst Particles: Hysteresis Effects. AIChE Journal, 37, 650-660. http://dx.doi.org/10.1002/aic.690370503

[6] Makinde, O.D. (2004) Exothermic Explosions in a Slab: A Case Study of Series Summation Technique. International Communications in Heat and Mass Transfer, 31, 1227-1231. http://dx.doi.org/10.1016/j.icheatmasstransfer.2004.08.020

[7] Makinde, O.D. (2005) Strong Exothermic Explosions in a Cylindrical Pipe: A Case Study of Series Summation Technique. Mechanics Research Communications, 32, 191-195. http://dx.doi.org/10.1016/j.mechrescom.2004.02.008

[8] Landau, L.D. and Lifshits, Y.M. (1964) Statistical Physics. Nauka, Moscow. (In Russian)

[9] Weisz, P.B. and Prater, C.D. (1954) Advances in Catalysis. Academic Press, New York, Vol. 6, p. 143.

[10] Wicke, E. and Brötz, W. (1949), Diffusion, Strömung und Reaktionsgeschwindigkeit im Innern poröser Kontaktkörper. Chemie Ingenieur Technik, 21, 219-226. http://dx.doi.org/10.1002/cite.330211104

[11] Aris, R. (1957) On Shape Factors for Irregular Particles-1: The Steady State Problem, Diffusion and Reaction. Chemical Engineering Sciences, 6, 262-268. http://dx.doi.org/10.1016/0009-2509(57)85028-3

[12] Weisz, P.B. and Goodwin, R.B. (1963) Combustion of Carbonaceous Deposits within Porous Catalyst Particles I, Diffusion-Controlled Kinetics. Journal of Catalyst, 2, 397-404. http://dx.doi.org/10.1016/0021-9517(63)90104-0

[13] Weisz, P.B. and Hicks, J.S. (1962) The Behaviour of Porous Catalyst Particles in View of Internal Mass and heat Diffusion Effects. Chemical Engineering Science, 17, 265-275. http://dx.doi.org/10.1016/0009-2509(62)85005-2 
[14] Adomian, G. (1984) Convergent Series Solution of Nonlinear Equations. Journal of Computational and Applied Mathematics, 11, 225-230. http://dx.doi.org/10.1016/0377-0427(84)90022-0

[15] Hasan, Y.Q. and Zhu, L.M. (2008) Modified Adomian Decomposition Method for Singular Initial Value Problems in the Second-Order Ordinary Differential Equations. Surveys in Mathematics and Its Applications, 3, 183-193.

[16] Hosseini, M.M. (2006) Adomian Decomposition Method with Chebyshev Polynomials. Applied Mathematics and Computation, 175, 1685-1693. http://dx.doi.org/10.1016/j.amc.2005.09.014

[17] Wazwaz, A.M. (1999) A Reliable Modification of Adomian Decomposition Method. Applied Mathematics and Computation, 102, 77-86. http://dx.doi.org/10.1016/S0096-3003(98)10024-3 


\section{Appendix A. Basic Concept of Modified Adomian Decomposition Method [16]}

Consider the nonlinear differential equation in the form

$$
y^{\prime \prime}+\frac{2 n}{x} y^{\prime}+\frac{n(n-1)}{x^{2}} y+F(x, y)=g(x) ; n \geq 0
$$

with initial condition

$$
y(0)=A, y^{\prime}(0)=B
$$

where $F(x, y)$ is a real function, $g(x)$ is the given function and $A$ and $B$ are constants. The differential operation is proposed as follows [17]

$$
L=x^{-n} \frac{\mathrm{d}^{2}}{\mathrm{~d} x^{2}} x^{n} y
$$

So, the problem (A.1) can be written as,

$$
L y=g(x)-F(x, y) .
$$

The inverse operator $L^{-1}$ is therefore considered a two-fold integral operator, as below.

$$
L^{-1}(.)=x^{-n} \int_{0}^{x} \int_{0}^{x} x^{n}(.) \mathrm{d} x \mathrm{~d} x
$$

Applying $L^{-1}$ of (A.5) to the first three terms $y^{\prime \prime}+\frac{2 n}{x} y^{\prime}+\frac{n(n-1)}{x^{2}} y$ of Equation (A.1) we find

$$
\begin{aligned}
L^{-1}\left(y^{\prime \prime}+\frac{2 n}{x} y^{\prime}+\frac{n(n-1)}{x^{2}} y\right) & =x^{-n} \int_{0}^{x} \int_{0}^{x} x^{n}\left(y^{\prime \prime}+\frac{2 n}{x} y^{\prime}+\frac{n(n-1)}{x^{2}} y\right) \mathrm{d} x \mathrm{~d} x \\
& =x^{-n} \int_{0}^{x}\left(x^{n} y^{\prime}+n x^{n-1} y\right) \mathrm{d} x=y-y(0)
\end{aligned}
$$

By operating $L^{-1}$ on (A.4), we have

$$
y(x)=A+L^{-1} g(x)-L^{-1} F(x, y)
$$

The Adomian decomposition method introduce the solution $y(x)$ and the nonlinear function $F(x, y)$ by infinity series

$$
\begin{aligned}
& y(x)=\sum_{n=0}^{\infty} y_{n}(x), \\
& F(x, y)=\sum_{n=0}^{\infty} A_{n}
\end{aligned}
$$

where the components $y_{n}(x)$ of the solution $y(x)$ will be determined recurrently and the Adomian polynomials $A_{n}$ of $F(x, y)$ are evaluated [22, 23, 25] using the formula

$$
A_{n}(x)=\left.\frac{1}{n !} \frac{\mathrm{d}^{n}}{\mathrm{~d} \lambda^{n}} N\left(\sum_{n=0}^{\infty}\left(\lambda^{n} y_{n}\right)\right)\right|_{\lambda=0}
$$

By substituting (A.8) and (A.9) into (A.7),

$$
\sum_{n=0}^{\infty} y_{n}(x)=A+L^{-1} g(x)-L^{-1} \sum_{n=0}^{\infty} A_{n}
$$

Through using the Adomian decomposition method, the components $y_{n}(x)$ can be determined as

$$
\begin{aligned}
& y_{0}(x)=A+L^{-1} g(x) \\
& y_{n+1}(x)=-L^{-1}\left(A_{n}\right), n \geq 0
\end{aligned}
$$


which gives

$$
\begin{aligned}
& y_{0}(x)=A+L^{-1} g(x) \\
& y_{1}(x)=-L^{-1}\left(A_{0}\right) \\
& y_{2}(x)=-L^{-1}\left(A_{1}\right) \\
& y_{3}(x)=-L^{-1}\left(A_{2}\right)
\end{aligned}
$$

From (A.9) and (A.12), we can determine the components $y_{n}(x)$, and hence the series solution of $y(x)$ in (A.7) can be immediately obtained.

\section{Appendix B: General Solution of Equation (1) Using the Adomian Decomposition Method}

In this appendix, we derive the general solution of nonlinear Equation (1) by using the Adomian decomposition method. We write the Equation (1) in the operator form,

$$
L(y)=\phi_{0}^{2} y \exp \left[\frac{\gamma \beta(1-y)}{1+\beta(1-y)}\right]
$$

where $L=x^{-1} \frac{\mathrm{d}^{2}}{\mathrm{~d} \rho^{2}} x$. Applying the inverse operator $L^{-1}()=.x^{-1} \int_{0}^{x} \int_{0}^{x} x(.) \mathrm{d} x \mathrm{~d} x$ on both sides of Eqn.

yields

$$
y(x)=A x+B+\phi_{0}^{2} L^{-1}\left(y \exp \left[\frac{\gamma \beta(1-y)}{1+\beta(1-y)}\right]\right)
$$

where $A$ and $B$ are the constants of integration. We let,

$$
y(x)=\sum_{n=0}^{\infty} y_{n}(x)
$$

and

$$
N[y(x)]=\sum_{n=0}^{\infty} A_{n}
$$

where

$$
N[y(x)]=\left(y \exp \left[\frac{\gamma \beta(1-y)}{1+\beta(1-y)}\right]\right)
$$

In view of Equations (B. 3 - B. 5), Equation (B. 2) gives

$$
\sum_{n=0}^{\infty} y_{n}(x)=A x+B+\phi_{0}^{2} L^{-1} \sum_{n=0}^{\infty} A_{n}
$$

We identify the zeroth component as

$$
y_{0}(x)=A x+B
$$

Using the boundary condition (4) we get,

$$
y_{0}=1
$$

and the remaining components can be obtained using the recurrence relation

$$
y_{n+1}(x)=\phi_{0}^{2} L^{-1} A_{n}, n \geq 0
$$

where $A_{n}$ are the Adomian polynomials of $y_{1}, y_{2}, \cdots, y_{n}$. We can obtain the first few $A_{n}$ as follows: 


$$
\begin{gathered}
A_{0}=N\left(y_{0}\right)=1 \\
A_{1}=\frac{\mathrm{d}}{\mathrm{d} \lambda}\left[N\left(y_{0}+\lambda y_{1}\right)\right]=\frac{\phi_{0}^{2}}{6}(1-\gamma \beta)\left(x^{2}-1\right)
\end{gathered}
$$

The remaining polynomials can be generated easily, and so,

$$
\begin{gathered}
y_{1}=\frac{\phi_{0}^{2}}{6}(1-\gamma \beta)\left(x^{2}-1\right) \\
y_{2}=\frac{7 \phi_{0}^{4}(1-\gamma \beta)}{360}+\phi_{0}^{4}(1-\gamma \beta)\left(\frac{x^{4}}{120}-\frac{x^{2}}{36}\right)
\end{gathered}
$$

Adding (B. 8), (B. 12) and (B. 13) we get the Equation (6) in the text.

\section{Appendix C: The Matlab Program to Find the Numerical Solution of Equation (1)}

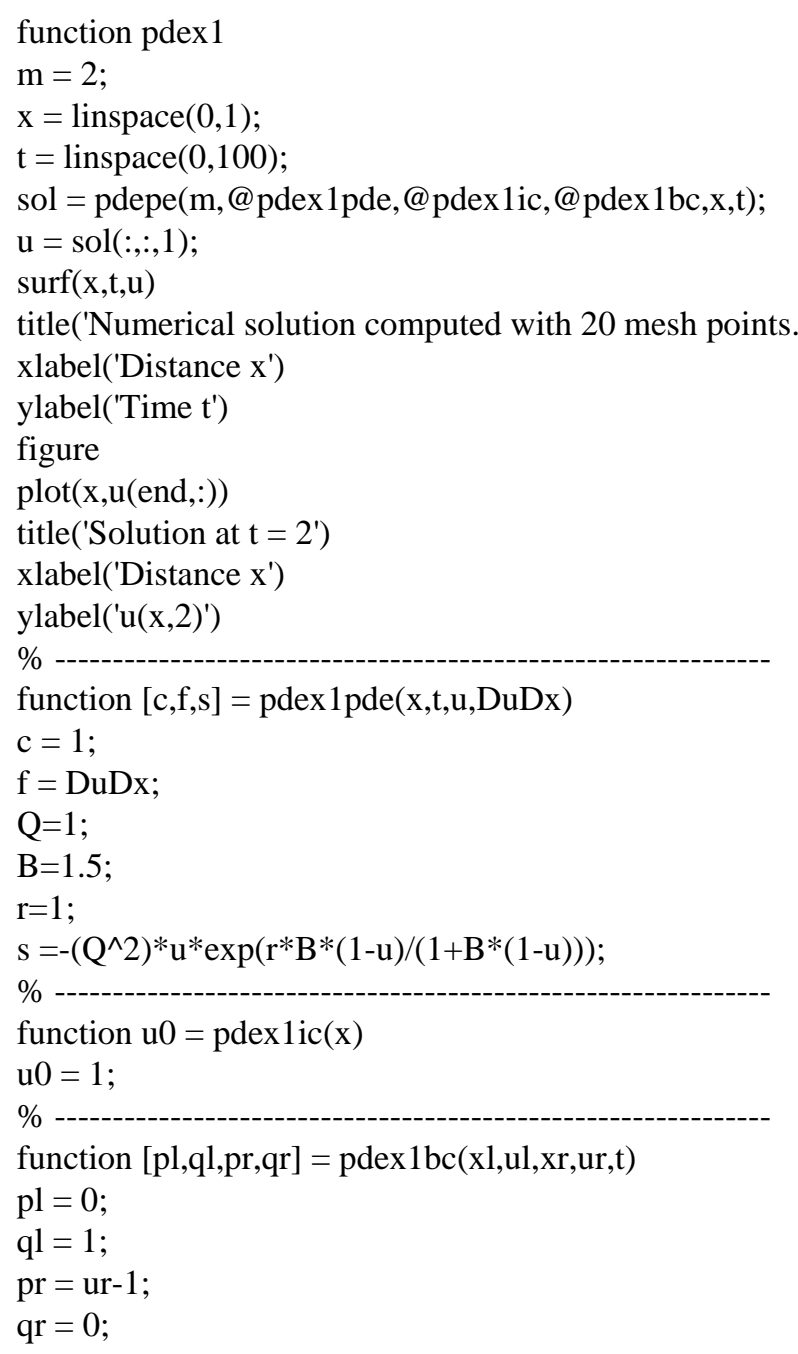

\section{Nomenclature}

$C_{\mathrm{A}}$ Concentration of reactant A inside the catalyst pellet $\left(\mathrm{mole} / \mathrm{cm}^{3}\right)$

$C_{\mathrm{A}, \mathrm{s}}$ Concentration of reactant A at the surface of catalyst pellet (mole/ $\left.\mathrm{cm}^{3}\right)$

$D_{\varepsilon} \quad$ Effective diffusivity inside the catalyst pellet $\left(\mathrm{cm}^{2} \cdot \mathrm{s}^{-1}\right)$ 


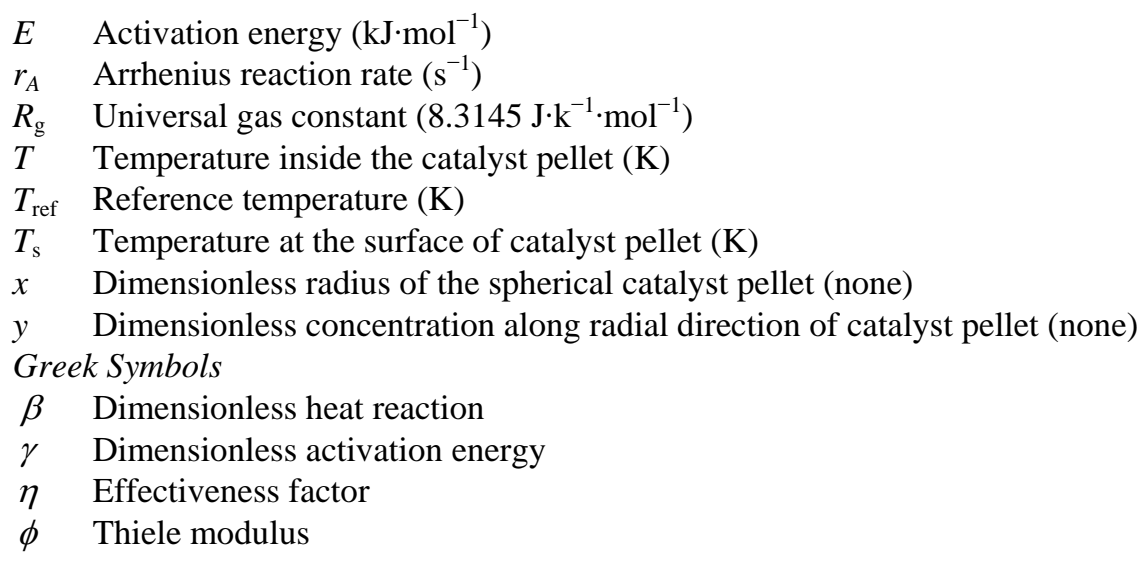

Submit or recommend next manuscript to SCIRP and we will provide best service for you:

Accepting pre-submission inquiries through Email, Facebook, Linkedin, Twitter, etc A wide selection of journals (inclusive of 9 subjects, more than 200 journals)

Providing a 24-hour high-quality service

User-friendly online submission system

Fair and swift peer-review system

Efficient typesetting and proofreading procedure

Display of the result of downloads and visits, as well as the number of cited articles

Maximum dissemination of your research work

Submit your manuscript at: http://papersubmission.scirp.org/ 